\title{
Directed evolution of Vibrio fischeri LuxR for increased sensitivity to a broad spectrum of acyl-homoserine lactones
}

Cynthia H. Collins, ${ }^{1}$ Frances H. Arnold ${ }^{1,2}$ and

Jared R. Leadbetter ${ }^{3 *}$

${ }^{1}$ Biochemistry and Molecular Biophysics, ${ }^{2}$ Division of Chemistry and Chemical Engineering, ${ }^{3}$ Environmental Science and Engineering, California Institute of Technology, Pasadena, CA 91125, USA.

\section{Summary}

LuxR-type transcriptional regulators play key roles in quorum-sensing systems that employ acylhomoserine lactones (acyl-HSLs) as signal molecules. These proteins mediate quorum control by changing their interactions with RNA polymerase and DNA in response to binding their cognate acyl-HSL. The evolutionarily related LuxR-type proteins exhibit considerable diversity in primary sequence and in their response to acyl-HSLs having acyl groups of differing length and composition. Little is known about which residues determine acyl-HSL specificity, and less about the evolutionary time scales required to forge new ones. To begin to examine such issues, we have focused on the LuxR protein from Vibrio fischeri, which activates gene transcription in response to binding its cognate quorum signal, 3oxohexanoyl-homoserine lactone (30C6HSL). Libraries of luxR mutants were screened for variants exhibiting increased gene activation in response to octanoyl-HSL (C8HSL), with which wild-type LuxR interacts only weakly. Eight LuxR variants were identified that showed a 100 -fold increase in sensitivity to C8HSL; these variants also displayed increased sensitivities to pentanoyl-HSL and tetradecanoyl-HSL, while maintaining a wild-type or greater response to $30 \mathrm{C} 6 \mathrm{HSL}$. The most sensitive variants activated gene transcription as strongly with C8HSL as the wild type did with 30C6HSL. With one exception, the amino acid residues involved were restricted to the $\mathrm{N}$-terminal, 'signal-binding' domain of LuxR. These residue positions differed from critical positions previously

Accepted 13 October, 2004. *For correspondence. E-mail jleadbetter@caltech.edu; Tel. (+1) 626395 4182; Fax (+1) 626 3952940.

C 2004 Blackwell Publishing Ltd identified via 'loss-of-function' mutagenesis. We have demonstrated that acyl-HSL-dependent quorumsensing systems can evolve rapidly to respond to new acyl-HSLs, suggesting that there may be an evolutionary advantage to maintaining such plasticity.

\section{Introduction}

Several mechanisms have evolved to allow diverse bacterial species to detect changes in their local population density, and to modulate their gene expression accordingly (Fuqua et al., 2001; Miller and Bassler, 2001). An opinion that quorum sensing is actually a form of diffusion sensing has recently been articulated (Redfield, 2002); however, it is the view of many that the two stances are not necessarily mutually exclusive. The population-density-based control of gene expression in bacteria has been termed quorum sensing (Fuqua et al., 1994). A paradigm of the quorum-sensing control of gene regulation within the Gram-negative phylum Proteobacteria has been the luminescence (lux) operon in Vibrio fischeri. In addition to the luciferase genes required for light production, this operon encodes LuxR, an acyl-homoserine lactone (acyl-HSL)-dependent transcriptional activator, and Luxl, an acyl-HSL synthase that catalyses the production of 3-oxohexanoyl-homoserine lactone (30C6HSL) (Engebrecht and Silverman, 1984). Each bacterium expresses the Lux proteins at low basal levels throughout its entire lifecycle. At low cell densities, the small amounts of the amphipathic 30C6HSL signal that are produced diffuse away from the cells (Kaplan and Greenberg, 1985; Pearson et al., 1999). However, as a local population increases in density, 30C6HSL concentrations increase. This results in a shift of the LuxR equilibrium towards its $30 \mathrm{C6HSL}-$ bound, active state. Acyl-HSL binding leads to dimerization of LuxR and binding to the lux box, a 20 base pair inverted repeat located in the $\mathrm{P}_{l u x}$ promoter (Egland and Greenberg, 1999). There the acyl-HSL-bound LuxR dimer activates expression of the lux genes after the recruitment of RNA polymerase (Stevens and Greenberg, 1997; Stevens et al., 1999).

To date, genes encoding more than 50 Luxl/LuxR pairs have been identified in diverse species belonging 
to the $\alpha-, \beta-$, and $\gamma$-subdivisions of the Proteobacteria (Lerat and Moran, 2004). While the basic quorum-sensing mechanism has been fairly well conserved in most species studied, the physiological functions controlled by acyl-HSL-based quorum-sensing systems are varied and include virulence (Passador et al., 1993; von Bodman and Farrand, 1995), antibiotic synthesis (Bainton et al., 1992; Pierson et al., 1994) and biofilm formation (Davies et al., 1998; Parsek and Greenberg, 1999). A LuxR homologue typically responds to an acyl-HSL synthesized by its partner Luxl homologue. Known quorum-sensing systems are tuned to preferentially synthesize, and respond to, a specific acyl-HSL having an acyl side group that may range in length from four to 16 carbons, may contain a degree of unsaturation, and may be modified with a carbonyl or hydroxyl functional group at carbon position 3 (Schaefer et al., 2000; 2002).

Are LuxR homologues evolutionarily pliable, i.e. able to move into new signalling niches on short time scales? We are interested in this issue and in identifying how the specificity of acyl-HSL response is determined. The ability of LuxR to regulate gene expression in response to an acyl-HSL depends on signal recognition and binding; it may also include acyl-HSL-specific dimerization and DNA-binding mechanisms. Because LuxR homologues generally share low amino acid sequence identities $(\leq 25 \%)$, however, it is difficult to determine precisely how they discriminate between various acyl-HSL molecules, or how this discrimination evolves and changes as a result of varying selective pressures. In a recent study on acyl-HSL-binding specificity, residues of TraR (a LuxR homologue) predicted to interact with the carbonyl moiety of its cognate signal, 3-oxooctanoyl-HSL (3OC8HSL) were mutated with the goal of stimulating the response of this protein to an analogue, octanoyl-HSL (C8HSL) (Chai and Winans, 2004). Rather than showing improved sensitivity to C8HSL, these variants lost the strong response that wild-type TraR displays towards $30 \mathrm{C} 8 \mathrm{HSL}$. The attempts of Chai and Winans to shift the specificity of TraR towards $30 \mathrm{C} 6 \mathrm{HSL}$ by introducing bulkier hydrophobic amino acids, and thereby reduce the size of the acyl binding site, also led, in all but one case, to variants with greatly decreased acyl-HSL affinities. Thus, alternative approaches may be more successful at altering the acyl-HSL specificities of LuxR or its homologues. To identify LuxR variants with altered acyl-HSL specificity, and to identify key residues that modulate this property, we have developed and implemented a system to direct the evolution of LuxR-mediated gene activation. Variants of LuxR were generated and screened to identify those that are highly responsive to acyl-HSL signals to which this transcriptional activator does not normally respond.

\section{Results}

\section{Cloning vectors and screening tools}

Modifications to a commercial two-plasmid system were made to allow for rapid cloning of luxR mutant libraries and screening for gene activation in response to exogenous signal molecules. The first plasmid, pLuxR, contains wild-type luxR under the control of a $\mathrm{P}_{\text {lac/ara-1 }}$ hybrid promoter. The luxR allele is constitutively expressed when placed in Escherichia coli strains lacking lacl and araC. The second plasmid, pluxGFPuv, contains the gene gfpuv placed under the control of the $\mathrm{P}_{\text {luxl }}$ promoter. The gfpuv gene encodes a green fluorescent protein variant, GFPuv, which becomes visible when excited with standard, long-wavelength ultraviolet light (Crameri et al., 1996). GFPuv expression from the $P_{\text {luxl }}$ promoter of pluxGFPuv is dependent upon the degree of activation that occurs as a result of a functional LuxR variant binding to a specific acyl-HSL signal molecule. The relative levels of gene activation observed with wild-type LuxR and the acyl-HSLs used in this study were similar to those previously reported in V. fischeri (Eberhard et al., 1986) and E. coli (Schaefer et al., 1996).

\section{First-generation laboratory evolution - error-prone PCR}

We used the two-plasmid system to identify LuxR variants that showed an increased ability to activate gene transcription in response to C8HSL. Past studies have shown that low-micromolar concentrations of $\mathrm{C} 8 \mathrm{HSL}$ were required to achieve the same level of gene expression achieved by $5 \mathrm{nM}$ of $30 \mathrm{C} 6 \mathrm{HSL}$, the cognate signal of LuxR (Eberhard et al., 1986). In the current system, a C8HSL concentration of approximately $2 \mu \mathrm{M}$ was required to achieve the level of gene activation obtained with $10 \mathrm{nM} 30 \mathrm{C} 6 \mathrm{HSL}$. Random mutations were introduced into wild-type luxR by error-prone PCR. E. coli hosts containing pluxGFPuv were transformed with pLuxR vectors containing the mutagenized PCR products. Transformants were initially plated onto LuriaBertani (LB)-agar amended with $50 \mathrm{nM} \mathrm{C8HSL}$ and antibiotics as appropriate. However, initial screening with this concentration of acyl-HSL yielded only one mutant, LuxR-G1A. Subsequently, the library was screened for activation with $200 \mathrm{nM}$ C8HSL. Colonies were screened under illumination with $365 \mathrm{~nm}$ ultraviolet light. Most colonies appeared dark under such illumination, indicating the expression of LuxR variants having either wild-type or non-functional properties. Of the $\sim 20000$ colonies that were screened, nine fluorescent colonies were identified. These nine colonies were isolated and verified by re-screening, which identified one as a false positive. The remaining eight alleles were amplified and cloned into fresh background plasmids and strains to ensure 
that the observed phenotype was due to changes within luxR. An additional false positive was identified by doing so.

Of the seven remaining alleles, two were shown to be identical by DNA sequence analysis. The six LuxR variants from this first generation of laboratory evolution were designated LuxR-G1A through LuxR-G1F (Fig. 1). Quantitative characterization of the response of these mutants to $30 \mathrm{C} 6 \mathrm{HSL}$ and $\mathrm{C} 8 \mathrm{HSL}$ was performed in liquid-phase assays by monitoring fluorescence levels using a microtiterplate spectrofluorimeter. As shown in Table 1, all six variants showed increased gene expression in the presence of $100 \mathrm{nM}$ C8HSL as compared to wild-type LuxR. Amino acid substitutions identified in the first generation are shown in Fig. 1.

\section{Second-generation laboratory evolution - DNA shuffling of mutant luxR alleles}

To investigate whether the mutations could be combined to yield further improvements in C8HSL response, a new library of luxR alleles was generated via DNA shuffling of the six alleles from the first generation, under conditions designed to minimize new point mutations (Joern, 2003). As with the first generation, transformants were screened for GFPuv production on LB-agar, in this case amended with $50 \mathrm{nM}$ C8HSL. Of 1200 colonies transformed with this library, 102 exhibited fluorescence. To identify those variants most sensitive to $\mathrm{C} 8 \mathrm{HSL}$, these

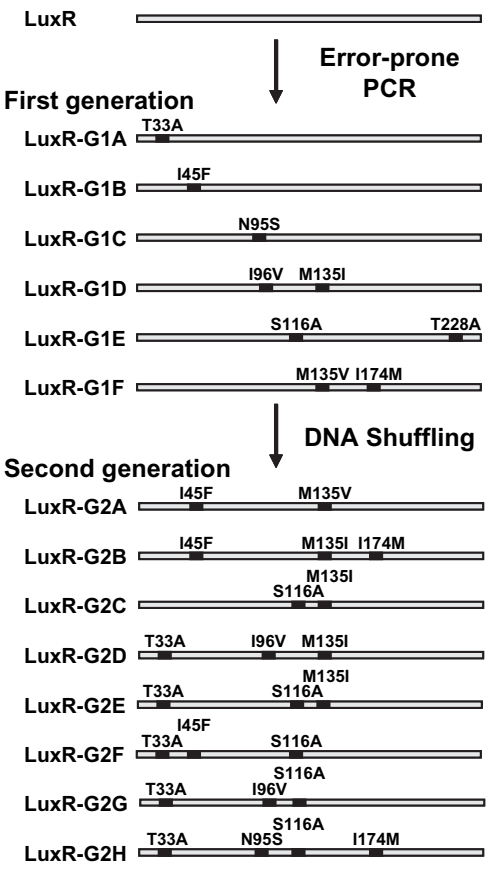

Fig. 1. Amino acid sequence changes to first- and second-generation LuxR variants showing increased gene activation in response to $\mathrm{C} 8 \mathrm{HSL}$. The six first-generation variants were identified from a library of approximately 20000 clones generated by error-prone PCR. Second-generation variants were generated by DNA shuffling of alleles from the first generation. Eight second-generation variants showing additional increases in gene activation in response to C8HSL were identified after screening 1200 clones. The positions of amino acid substitutions within the 250 amino acid LuxR protein are indicated as black rectangles (refer to Supplemental material Table S1 for nucleotide sequence changes).

Table 1. Activation of gfpuv gene expression by LuxR variants with four different acyl-HSLs.

\begin{tabular}{|c|c|c|c|c|c|c|c|}
\hline \multirow[b]{3}{*}{ LuxR type } & \multicolumn{7}{|c|}{ Fluorescence $(\mathrm{AU})^{\mathrm{a}}$} \\
\hline & \multirow{2}{*}{$\begin{array}{l}\text { No } \\
\text { acyl-HSL }\end{array}$} & \multicolumn{2}{|c|}{$30 \mathrm{C} 6 \mathrm{HSL}$} & \multicolumn{2}{|c|}{ C8HSL } & \multirow{2}{*}{$\frac{\mathrm{C} 5 \mathrm{HSL}}{100 \mathrm{nM}}$} & \multirow{2}{*}{$\frac{\mathrm{C} 14 \mathrm{HSL}}{500 \mathrm{nM}}$} \\
\hline & & $10 \mathrm{nM}$ & $100 \mathrm{nM}$ & $10 \mathrm{nM}$ & $100 \mathrm{nM}$ & & \\
\hline LuxR wt & $<\underline{50}$ & $\underline{1400}$ & $\underline{3000}$ & $<\underline{50}$ & $\underline{100}$ & $<\underline{50}$ & $<\underline{50}$ \\
\hline $\begin{array}{l}\text { First generation } \\
\text { LuxR-G1A }\end{array}$ & $<50$ & 2200 & 3300 & $<50$ & 800 & 100 & $<50$ \\
\hline LuxR-G1B & $<50$ & 2600 & 3200 & $<50$ & 800 & 500 & $<50$ \\
\hline LuxR-G1C & $<50$ & 2100 & 3000 & $<50$ & 700 & $<50$ & $<50$ \\
\hline LuxR-G1D & $<50$ & 400 & 2000 & 100 & 1500 & 700 & 200 \\
\hline LuxR-G1E & $<50$ & 2200 & 3200 & $<50$ & 500 & $<50$ & 100 \\
\hline LuxR-G1F & $<50$ & 600 & 2700 & $<50$ & 700 & 600 & 100 \\
\hline \multicolumn{8}{|c|}{ Second generation } \\
\hline LuxR-G2A & $<50$ & 1600 & 3200 & 200 & 1700 & 2200 & 100 \\
\hline LuxR-G2B & $<50$ & 2400 & 3500 & 900 & 2900 & 3000 & 400 \\
\hline LuxR-G2C & $<50$ & 500 & 1900 & 200 & 1900 & 1000 & 700 \\
\hline LuxR-G2D & $<50$ & 1300 & 3200 & 800 & 3100 & 2300 & 1300 \\
\hline LuxR-G2E & $<50$ & 1400 & 3200 & 1100 & 3600 & 3200 & 2200 \\
\hline LuxR-G2F & $<50$ & 3100 & 2900 & 800 & 2900 & 2600 & 900 \\
\hline LuxR-G2G & $<50$ & 3300 & 3500 & 500 & 2800 & 1400 & 1500 \\
\hline LuxR-G2H & $<50$ & 3300 & 3400 & 900 & 2500 & 1200 & 1500 \\
\hline
\end{tabular}

a. Units of fluorescence represent the fluorescence because of GFPuv production obtained with a given LuxR variant and pluxGFPuv. Fluorescence values were corrected with the background fluorescence obtained without a LuxR expressing plasmid. Both values were normalized to their cell densities prior to correction. All values are the mean of triplicate measurements. Variation was less than $\pm 15 \%$ for all reported values. Arbitrary fluorescence units (AU) represent the fluorescence due to GFPuv production. 
102 strains were screened using 10 and 20 nM C8HSL. Ten exhibited fluorescence under these conditions and were picked for further characterization. One false positive was identified after re-amplification and re-cloning of the alleles into fresh background materials. Sequence analysis revealed two of the alleles to be identical. Quantitative liquid-phase characterization of the eight remaining mutants showed that all exhibited remarkable increases in fluorescence output in response to not only $100 \mathrm{nM}$ but also $10 \mathrm{nM}$ C8HSL, as compared to either wild-type LuxR or any of the first-generation LuxR variants (Table 1). Sequencing revealed that no additional residue positions had been changed; the second-generation sequences were all recombinants of the parent sequences (Fig. 1).

\section{C8HSL and 30C6HSL dose responses}

We compared the influence of a range of $30 \mathrm{C} 6 \mathrm{HSL}$ and C8HSL concentrations on transcriptional activation by wild-type LuxR and each of the second-generation variants (Fig. 2). Wild-type LuxR required approximately $2 \mu \mathrm{M}$ $\mathrm{C} 8 \mathrm{HSL}$ to elicit half the maximum GFPuv fluorescence observed using saturating amounts of $30 \mathrm{C} 6 \mathrm{HSL}$, whereas only $10 \mathrm{nM} 30 \mathrm{C} 6 \mathrm{HSL}$ was required for half-maximal activation (Fig. 2A). In comparison, all second-generation LuxR variants showed an increased sensitivity to C8HSL, requiring only $15-50 \mathrm{nM}$ to achieve the half-maximal 30C6HSL-induced fluorescence of the wild type. Thus, C8HSL sensitivity and response by these LuxR variants increased by 20 - to 100 -fold. Variants LuxR-G2D and LuxR-G2E responded as sensitively and strongly to C8HSL as wild-type LuxR did to 30C6HSL (Fig. 2E and $\mathrm{F})$. The acquisition of this trait had little or no deleterious impact on the sensitivity of these variants to $30 \mathrm{C} 6 \mathrm{HSL}$. Three variants, LuxR-G2A, LuxR-G2D and LuxR-G2E (Fig. 2B, E and F), showed responses to 30C6HSL indistinguishable from that of wild-type LuxR. Variants LuxRG2F, LuxR-G2G and LuxR-G2H (Fig. 2G-I) showed fivefold increased sensitivity to $30 \mathrm{C} 6 \mathrm{HSL}$, exhibiting signifi-
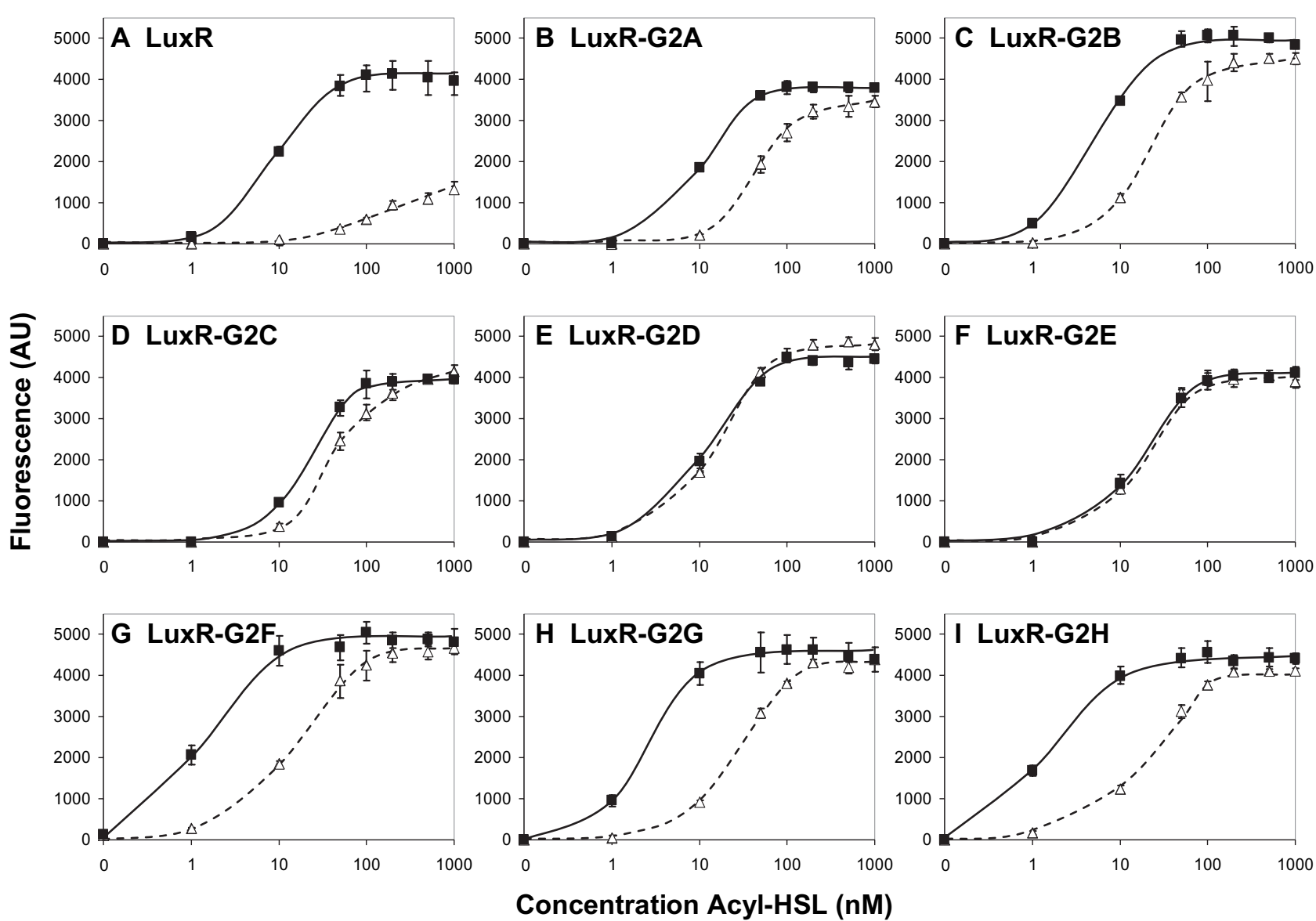

Fig. 2. Detection of C8HSL and 30C6HSL by wild-type LuxR and eight second-generation LuxR variants. For each panel and variant (A-I), data for $30 \mathrm{C} 6 \mathrm{HSL}$ are represented by closed squares $(\square)$, data for C8HSL are represented by open triangles $(\triangle)$ ). Fluorescence data reported are normalized to optical density, and corrected by subtracting background fluorescence from a control strain carrying pPROLar.A122 with pluxGFPuv. All measurements were performed in triplicate. Error bars indicate either the range of values or the standard deviation, whichever was greater. 


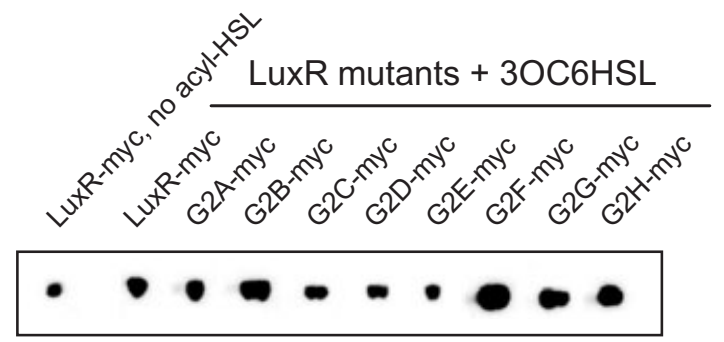

Fig. 3. Protein concentrations of second-generation LuxR variants. The immunoblot was performed on a SDS-PAGE display of the total proteins harvested from strains expressing $c$-myc tagged secondgeneration LuxR variants incubated in the presence of $1 \mu \mathrm{M}$ $30 \mathrm{C} 6 \mathrm{HSL}$. myc-coupled fluorescence for each of the variants varied by less than $10 \%$ in the presence and absence of acyl-HSL (latter not presented). In the absence of acyl-HSL, the amount of LuxR protein detected for each variant was within twofold of that for the wild-type LuxR-myc fusion.

cant levels of fluorescence with concentrations as low as $1 \mathrm{nM}$.

Increased sensitivity to 30C6HSL can be attributed to increases in LuxR protein concentration

To investigate whether increases in expression were responsible for observed increases in gene activation in response to $30 \mathrm{C} 6 \mathrm{HSL}$, concentrations of the variant LuxR proteins were measured. Fusions of the $c$-myc epitope to the C-termini of wild-type LuxR and each of the secondgeneration variants were constructed. All c-myc LuxR variants functioned well, i.e. exhibited responses to acylHSLs which were similar to the variant lacking the c-myc epitope. Protein concentrations were monitored under different incubation conditions by Western immunoblot assay and compared.

We tested the accumulation of wild-type LuxR and the second-generation variant proteins after incubation in the absence or presence of saturating amounts of $30 \mathrm{C} 6 \mathrm{HSL}$. In the absence of acyl-HSL, the amount of protein detected by Western immunoblot for each of the variants differed by less than twofold from wild-type LuxR; however, in the presence of $1 \mu \mathrm{M} 30 \mathrm{C} 6 \mathrm{HSL}$, the four variants with increased responses to 30C6HSL, LuxR-G2B, -G2F, -G2G and $-\mathrm{G} 2 \mathrm{H}$, also showed increased amounts of protein as compared to wild type (Fig. 3). Thus mutations leading to higher LuxR levels may lead to the increased sensitivities of these variants to $30 \mathrm{C} 6 \mathrm{HSL}$ and, in part, C8HSL.

The amount of wild-type LuxR detected in the presence of $30 \mathrm{C} 6 \mathrm{HSL}$ was approximately twofold more than was detected in the absence of signal. The increase in protein levels observed with the addition of $30 \mathrm{C6HSL}$ is similar to that observed for TraR (Chai and Winans, 2004), consistent with the notion that acyl-HSL binding enhances the folding and/or stability of LuxR. If so, acyl-HSL binding and protein stability are inextricably linked.
Response of LuxR variants to acyl-HSLs with long and short acyl chains

The ability of the C8HSL-responsive LuxR variants to activate gene expression with other acyl-HSLs was also investigated. The response of each variant was examined using $100 \mathrm{nM}$ pentanoyl-homoserine lactone (C5HSL) and $500 \mathrm{nM}$ tetradecanoyl-homoserine lactone (C14HSL), two acyl-HSLs to which wild-type LuxR exhibits essentially no response (Table 1). Four first-generation variants (LuxR-G1A, -G1B, -G1D and -G1F) showed small, yet significant, responses to C5HSL, as did three (LuxR-G1D, -G1E and -G1F) to C14HSL.

The second-generation variants all showed marked improvements in their sensitivity and response to both C5HSL and C14HSL. LuxR-G2A showed a threefold and LuxR-G2B a >fourfold improvement in sensing C5HSL when compared to the best of the first-generation mutants. LuxR-G2G and LuxR-G2H showed a > sevenfold increase in gene activation in the presence of $\mathrm{C} 14 \mathrm{HSL}$ when compared to the best of the first-generation mutants. LuxR-G2D, -G2E and -G2F all showed broadened acyl-HSL specificity, exhibiting strong responses to both C5HSL and C14HSL. The most responsive mutant, LuxR-G2E, showed a fivefold increase in fluorescence with $\mathrm{C} 5 \mathrm{HSL}$ and an 11-fold increase with $\mathrm{C} 14 \mathrm{HSL}$, while maintaining a wild-type level of response to $30 \mathrm{C} 6 \mathrm{HSL}$.

\section{Acyl-HSL specificity of the second-generation variants}

The ratio of the $30 \mathrm{C} 6 \mathrm{HSL}$ and $\mathrm{C} 8 \mathrm{HSL}$ concentrations required to attain half-maximal gene expression, termed the specificity, $\mathrm{S}$, was used to assess changes in acyl-HSL responses (Table 2). While wild-type LuxR has an $S$ of 200 , indicating that it requires 200 -fold more C8HSL than $30 \mathrm{C} 6 \mathrm{HSL}$ to reach half-maximal fluorescence levels, all of the second-generation mutants have $S<20$. The variant with the largest increase in response to C8HSL, LuxR-

Table 2. Specificity of second-generation LuxR variants.

\begin{tabular}{lccc}
\hline LuxR type & {$[30 \mathrm{C} 6 \mathrm{HSL}]_{50}(\mathrm{nM})^{\mathrm{a}}$} & {$[\mathrm{C} 8 \mathrm{HSL}]_{50}(\mathrm{nM})^{\mathrm{a}}$} & $\mathrm{S}^{\mathrm{b}}$ \\
\hline LuxR wt & 10 & 2000 & 200 \\
LuxR-G2A & 10 & 50 & 5.0 \\
LuxR-G2B & 5 & 20 & 4.0 \\
LuxR-G2C & 20 & 35 & 1.8 \\
LuxR-G2D & 10 & 15 & 1.5 \\
LuxR-G2E & 20 & 20 & 1.0 \\
LuxR-G2F & 1.5 & 20 & 13.3 \\
LuxR-G2G & 2.5 & 30 & 12.0 \\
LuxR-G2H & 1.5 & 25 & 16.7
\end{tabular}

a. $[30 \mathrm{OC} 6 \mathrm{HSL}]_{50}$ and $[\mathrm{C} 8 \mathrm{HSL}]_{50}$ are the concentrations of these acylHSLs required to reach half-maximal gene activation as measured from the fluorescence output of GFPuv.

b. The specificity constant, S, corresponds to the ratio of the concentrations of $30 \mathrm{C} 6 \mathrm{HSL}$ and $\mathrm{C} 8 \mathrm{HSL}$ required to achieve half-maximal gene activation, or $[3 \mathrm{OC} 6 \mathrm{HSL}]_{50} /[\mathrm{C} 8 \mathrm{HSL}]_{50}$. 
G2E, has $S=1$ : it responds equally to both acyl-HSLs. This variant is also sensitive to C5HSL and C14HSL (see above). The variants which showed increased protein concentrations (LuxR-G2F, -G2G and -G2H) also exhibited a $>10$-fold decrease in $S$ as compared to wild type. Whereas increases in protein concentration can increase response to all acyl-HSLs, they can not account for changes in specificity. Acyl-HSL specificity has been significantly altered in all of the variants.

\section{Single-mutants identify six mutations that increase C8HSL response by LuxR}

Because every first-generation luxR mutant contained more than one nucleotide substitution, further information is required to determine which lead to increased response to C8HSL. Single-mutants with each of the 13 nucleotide substitutions identified in the six first-generation variants, including four synonymous mutations, were prepared using site-directed mutagenesis. Quantitative liquid-phase characterization showed that amino acid substitutions at positions 33, 45, 95, 116, 135 and 174 confer increases in sensitivity to C8HSL (Table 3). Fluorescence levels similar to those obtained with wild-type LuxR were found for substitutions at amino acid position 228 and synonymous mutations at nucleotide positions 162, 414 and 501. Two mutations, one leading to substitution of 196 by valine and the synonymous mutation at nucleotide position 159, are slightly deleterious, causing small decreases in gene activation. The single-mutant containing only the T33 to alanine substitution was more sensitive to C8HSL than LuxRG1A which contains both the T33A substitution and the synonymous mutation at position 159. However, the response of LuxR-G1D, which contains both the I96V and M135I substitutions, was indistinguishable from the single-mutant containing only the M135I mutation, suggesting that the deleterious effects of the $196 \mathrm{~V}$ mutation may be masked by the presence of a beneficial substitution

\section{Discussion}

The results of this study indicate that LuxR can evolve rapidly to sense and respond to a diversity of acyl-HSL signal molecules. By screening for changes in gene activation in the presence of C8HSL, we were able to identify variants of this signal-dependent transcriptional activator that respond strongly to this molecule and two additional acyl-HSLs. Only two generations and three or fewer residue changes were required to generate LuxR variants that activate gene expression in response to $\mathrm{C} 8 \mathrm{HSL}$ with sensitivity equal to its response to the natural signal, 30C6HSL.

The variants identified in this study may be altered in more than one of multiple properties and processes involved in gene activation by LuxR. LuxR-mediated gene activation involves key events of signal binding, dimerization, conformational changes, DNA binding, and transcriptional activation via interactions with RNA polymerase and other proteins (Fuqua et al., 2001). Changes to LuxR stability (in the presence and absence of a competent acylHSL molecule), folding, solubility and expression could also affect its ability to activate genes. Indeed, the stability of TraR (a LuxR homologue) in the cytosol has been

Table 3. Activation of gfpuv gene expression by luxR single mutants.

\begin{tabular}{|c|c|c|c|c|c|c|}
\hline \multirow{3}{*}{$\begin{array}{l}\text { Nucleotide } \\
\text { substitution }\end{array}$} & \multirow{3}{*}{$\begin{array}{l}\text { Amino acid } \\
\text { substitution }\end{array}$} & \multicolumn{5}{|c|}{ Fluorescence $(\mathrm{AU})^{\mathrm{a}}$} \\
\hline & & \multirow{2}{*}{$\begin{array}{l}\text { No } \\
\text { acyl-HSL }\end{array}$} & \multicolumn{2}{|c|}{$30 \mathrm{C} 6 \mathrm{HSL}$} & \multicolumn{2}{|c|}{$\mathrm{C} 8 \mathrm{HSL}$} \\
\hline & & & $10 \mathrm{nM}$ & $100 \mathrm{nM}$ & $10 \mathrm{nM}$ & $100 \mathrm{nM}$ \\
\hline luxR wt & LuxR wt & $<\underline{50}$ & 1700 & 3000 & $<\underline{50}$ & $\underline{200}$ \\
\hline $\mathrm{A} 97 \rightarrow \mathrm{G}$ & Thr33 $\rightarrow$ Ala & $<50$ & 2500 & 3000 & 200 & 1200 \\
\hline $\mathrm{A} 133 \rightarrow \mathrm{T}$ & lle $45 \rightarrow$ Phe & $<50$ & 3100 & 3100 & 300 & 1400 \\
\hline $\mathrm{A} 159 \rightarrow \mathrm{G}$ & Syn $^{b}$ & $<50$ & 300 & 1000 & $<50$ & $<50$ \\
\hline $\mathrm{T} 162 \rightarrow \mathrm{A}$ & Syn & $<50$ & 2000 & 2800 & 100 & 500 \\
\hline $\mathrm{A} 284 \rightarrow \mathrm{G}$ & Asn95 $\rightarrow$ Ser & $<50$ & 2300 & 2600 & 200 & 1000 \\
\hline $\mathrm{A} 286 \rightarrow \mathrm{G}$ & $\mathrm{Ile} 96 \rightarrow$ Val & $<50$ & 900 & 1200 & $<50$ & 200 \\
\hline $\mathrm{A} 346 \rightarrow \mathrm{G}$ & Ser116 $\rightarrow$ Ala & $<50$ & 2600 & 3000 & 200 & 700 \\
\hline $\mathrm{A} 403 \rightarrow \mathrm{G}$ & Met135 $\rightarrow$ Val & $<50$ & 900 & 2100 & 100 & 700 \\
\hline $\mathrm{G} 405 \rightarrow \mathrm{A}$ & Met135 $\rightarrow \| l e$ & $<50$ & 700 & 1800 & 200 & 1100 \\
\hline $\mathrm{A} 414 \rightarrow \mathrm{C}$ & Syn & $<50$ & 1300 & 2700 & $<50$ & 200 \\
\hline $\mathrm{T} 501 \rightarrow \mathrm{C}$ & Syn & $<50$ & 2000 & 2600 & 100 & 400 \\
\hline $\mathrm{A} 522 \rightarrow \mathrm{G}$ & Ile174 $\rightarrow$ Met & $<50$ & 2300 & 2800 & 100 & 700 \\
\hline $\mathrm{A} 683 \rightarrow \mathrm{G}$ & Thr228 $\rightarrow$ Ala & $<50$ & 1800 & 2400 & 100 & 300 \\
\hline
\end{tabular}

a. Units of fluorescence represent the fluorescence because of GFPuv production obtained with a given LuxR variant and p/uxGFPuv as described for Table 1.

b. Syn denotes a synonymous mutation, i.e. a nucleotide change which does not encode an amino acid change.

Arbitrary fluorescence units $(\mathrm{AU})$ represent the fluorescence due to GFPuv production. 
shown to increase dramatically upon binding a competent acyl-HSL (Zhu and Winans, 2001; Zhang et al., 2002). The increase in LuxR concentration observed in the presence of $30 \mathrm{C} 6 \mathrm{HSL}$, as compared to its concentration without signal, indicates that, like TraR, the stability of LuxR is affected by the presence of acyl-HSL. General improvements in response to various acyl-HSLs could result from changes in many of these properties. Acyl-HSL-specific response, however, can be extricated from these other properties by looking at $\mathrm{S}$, the ratio of responses, i.e. between the cognate and another acyl-HSL. Using this measure we have shown that the response to C8HSL was enhanced in all of the variants relative to the changes in response to $30 \mathrm{C} 6 \mathrm{HSL}$, suggesting key changes had occurred beyond general concentration effects, and likely at the level of acyl-HSL binding.

Past studies involving the mutagenesis of luxR have been instrumental in constructing a structural and functional map of LuxR (reviewed in Stevens and Greenberg, 1999). Regions of LuxR that are essential for its function were identified by screening random point mutations within luxR (Shadel et al., 1990; Slock et al., 1990). Mutations that led to non-functional LuxR variants clustered between residues 79-127 and 184-230. Reversal of the loss-offunction phenotype for several mutations within the $\mathrm{N}$ terminal critical region was achieved by adding high concentrations of $30 \mathrm{C} 6 \mathrm{HSL}$, suggesting that such mutations weaken the interaction between the sensor protein and its cognate signal (Shadel et al., 1990). None of the residue positions identified here were shown to be critical for function in the previous studies. Four of the mutations (T33A, I44F, M135I and M135V) are located outside of the region previously postulated to be involved in acyl-HSL binding, albeit well within the predicted N-terminal domain (Stevens and Greenberg, 1999). Thus, by evolving LuxR to respond to an acyl-HSL molecule to which it is normally insensitive, we have identified residues involved in acyl-HSL specificity and response that are distinct from those revealed during loss-of-function mutant studies (see Supplementary material, Fig. S1). This was not entirely unexpected, as many laboratory evolution studies have demonstrated that amino acids involved in modulating function are generally distinct from, and more tolerant to change, than those required for folding or function (Voigt et al., 2001).

The T33A, I45F, N95S, S116A, M135I and I174M mutations increase C8HSL response by LuxR

Analysis of the 13 single-site mutants showed that a single mutation from each of the first-generation variants was responsible for the observed increase in C8HSL response (Table 3), with the exception of LuxR-G1F, in which both the M135V and I174M mutations increase C8HSL sensitivity. Recombining these mutations led to LuxR variants with further increases in their ability to activate gene expression with $\mathrm{C} 8 \mathrm{HSL}$ but with varying responses to 30C6HSL, C5HSL and C14HSL. The absence of N95S in the second generation may be due to random chance, bias in the construction of the library, or its inability to confer any additional advantage when combined with the other mutations.

\section{Substitutions at position 135 likely stabilize interactions with straight-chain acyl-HSLs}

The variants which showed increased response to C8HSL with minimal changes in their response to $30 \mathrm{C} 6 \mathrm{HSL}$ (LuxR-G2A, -G2B, -G2C, -G2D and -G2E) all contain a substitution at position 135 (Fig. 2 and Table 1). Mutation of M135 to isoleucine or valine appears to stabilize interactions between LuxR and C8HSL without affecting the sensor's recognition of its cognate signal, 30C6HSL. Alignments of LuxR with a number of its homologues revealed that proteins that respond to acyl-HSLs containing a carbonyl group at the third carbon of their acyl chain often have a methionine residue at this position, whereas those that recognize straight-chain acyl-HSLs usually contain an isoleucine or a valine residue (see Supplementary material, Fig. S2). Thus natural and laboratory evolution appear to converge to the same answer. The predominant preference at this position in all LuxR homologues seems to be for a hydrophobic residue (isoleucine, valine, leucine or methionine). Alignment to the published structure of TraR shows that 135 is only two positions away from an amino acid required for recognition of the 3-oxo moiety of 3OC8HSL in TraR (Chai and Winans, 2004), and could potentially be involved in modulating the response to acylHSLs lacking a 3-oxo moiety. It also remains possible that residue 135 does make contact with the acyl-HSL molecule: the structure of LuxR has not yet been determined and may differ significantly from that of its distantly related homologue, TraR (Urbanowski et al., 2004).

\section{Acyl-HSL specificity is influenced by mutations at LuxR positions 33 and 45}

Each of the second-generation variants that exhibited the greatest increases in gene expression in response to C14HSL (LuxR-G2D to -G2H) contained the T33A mutation (Table 1). The T33A mutation may help LuxR to accommodate acyl-HSLs with acyl chains more than six carbons in length. TraR and LasR, which respond to acylHSLs having acyl side chains eight and 12 carbons in length, both contain an alanine residue at this position (Gambello and Iglewski, 1991; Fuqua and Winans, 1994). Substitution of 145 by phenylalanine, on the other hand, led to an increase in GFPuv production in response to $\mathrm{C} 5 \mathrm{HSL}$ in each of the three second-generation variants, 
LuxR-G2A, -G2B and -G2F, in which it was identified. Therefore, I45F might improve interactions with shortchain acyl-HSLs such as C5HSL.

The identification of four LuxR variants showing increased sensitivity to $30 \mathrm{C} 6 \mathrm{HSL}$ was unexpected, inasmuch as wild type already responds strongly to low nanomolar concentrations of this, its cognate signal. Increases in variant protein levels, as observed in the Western blots (Fig. 3), because of increases in expression, stability or solubility are the most likely explanation. The crystal structure of TraR was used to map the hypothetical positions of the amino acid substitutions identified in this study (Fig. 4). Curiously, the TraR residues which align with LuxR positions 145 and I174 are located at the interface between its signal- and DNA-binding domains. These mutations might act by stabilizing interactions between these two domains required for LuxR's switch-like behaviour, thereby conferring increases in gene activation.

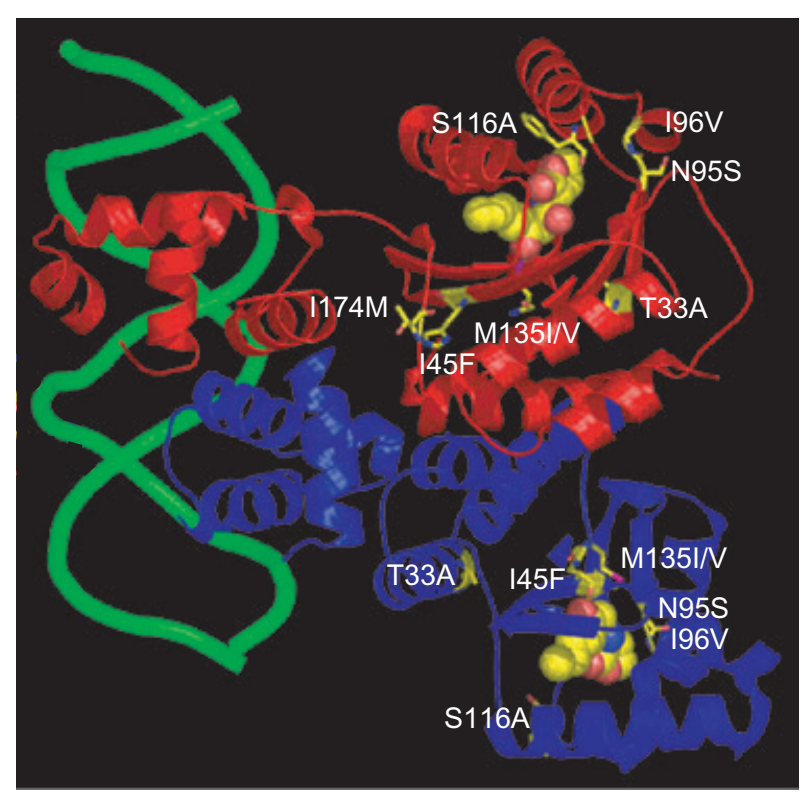

Fig. 4. Hypothetical positions of residues in TraR corresponding to those found to modulate acyl-HSL specificity in LuxR. The crystal structure of the LuxR homologue TraR (PDB 1L3L) has been determined (Zhang et al., 2002). The two peptides comprising a TraR dimer are shown in red and blue. The double-stranded DNA to which TraR binds is shown in green. The two 3-oxooctanoyl-homoserine lactone (3OC8HSL) molecules are shown using space-filling spherical atoms (yellow represents carbon, red represents nitrogen, and blue represents oxygen). The amino acid residues which align with those identified in the LuxR variants exhibiting altered acyl-HSL responses are shown as sticks with colouring similar to that used for $30 \mathrm{C} 8 \mathrm{HSL}$. The amino acids identified occur predominantly within the $\mathrm{N}$-terminal domain and do not appear to make direct contact with the signal molecule. Because LuxR and TraR share less than $20 \%$ sequence identity at the amino acid level, these assignments are purely hypothetical. However, the overall structures of the two homologues are expected to be in large part similar. Their alignment can provide a useful first approximation of residue location within the threedimensional structure of any LuxR-type protein.
The acyl-HSL sensitivity of LuxR homologues may not have been maximized during natural evolution. LuxR-type proteins may be tunable over short evolutionary periods to meet the physical and chemical nature of the habitats in which the cells encoding them are active. Indeed, the amount of acyl-HSLs that must accumulate for a given quorum-sensing species to exhibit a quorum response can vary widely from low nanomolar to micromolar concentrations (Engebrecht and Silverman, 1984; Eberhard et al., 1986; Fuqua and Winans, 1994; von Bodman and Farrand, 1995; Whiteley et al., 1999; Chugani et al., 2001), and likely reflects the particular physiological ecology of the organism.

While this study indicates that LuxR can rapidly evolve to respond to a broad range of acyl-HSL molecules, it did not reveal which or how many evolutionary changes are required to refocus it to a new signal molecule. Our findings indicate that the easiest evolutionary solution to generating a protein that responds to a new signalling molecule is broadening its specificity. This is consistent with other directed evolution work that has shown that refocusing binding to shift, rather than broaden, specificity often requires additional evolutionary work (Matsumura and Ellington, 2001). Natural evolution of signal and substrate specificity has also been shown to fluctuate between specialized states through broad-specificity intermediates, allowing for the acquisition of new functions by expanding the capabilities of 'old' proteins (Waley, 1969; Ycas, 1974; Jenson, 1976; Kacser and Beeby, 1984; Lazcano et al., 1994). Laboratory evolution experiments which have successfully refocused binding or substrate specificity to a new target have specifically included screening for activity with a new substrate and the absence of activity with the enzyme's natural substrate (Santoro and Schultz, 2002; Voziyanov et al., 2002). In future laboratory evolution studies with LuxR, we hope to understand the basis for acyl-HSL sensing as well as how diverse acyl-HSL-based intercellular communications systems have evolved and continue to evolve.

\section{Experimental procedures}

\section{Bacterial strains, media and growth conditions}

The bacterial strains used in this study were: E. coli strain $\mathrm{DH} 5 \alpha\left(\mathrm{F}^{-} \phi 80 \mathrm{dLacZ} \Delta \mathrm{M} 15 \Delta\right.$ (lacZYA-argF) U169 deoR recA1 endA1 $h s d \mathrm{R} 17\left(\mathrm{r}_{\mathrm{k}}^{-}, \mathrm{m}_{\mathrm{k}}{ }^{+}\right)$phoA supE44 $\lambda$ - thi-1 gyrA96 relA1) and E. coli DH5 $\alpha$ containing pluxGFPuv [DH5 $\alpha$ (p/uxGFPuv)], a pPROTet.E133-derived LuxR/acyl-HSL-inducible GFPuv expression vector encoding chloramphenicol resistance. Competent $\mathrm{DH} 5 \alpha$ and $\mathrm{DH} 5 \alpha($ pluxGFPuv) cells were prepared by using the Z-competent E. coli Transformation Kit (Zymo Research, Orange, CA). E. coli strains were cultured at $37^{\circ} \mathrm{C}$ in LB medium or on LB-agar plates. Antibiotics were added at the following concentrations: $100 \mu \mathrm{g} \mathrm{m}^{-1}$ chloramphenicol, or $20 \mu \mathrm{g} \mathrm{ml}^{-1}$ kanamycin. Acyl-HSL stock solutions 
of 1 and $10 \mathrm{mM}$ were prepared by dissolving appropriate amounts in ethyl acetate acidified with glacial acetic acid $(0.01 \% \mathrm{v} / \mathrm{v})$, and stored at $-20^{\circ} \mathrm{C}$. Acyl-HSLs used in these studies were: $30 \mathrm{C6HSL}$ (Sigma Aldrich, St Louis, MO), C5HSL (B. Hauer), C8HSL (Fluka, St Louis, MO) and C14HSL (Fluka). For screening experiments, C8HSL was amended to LB-agar media prior to solidification to ensure complete mixing. For liquid-phase experiments, acyl-HSL was dispensed into sterile tubes, the ethyl acetate was evaporated under a stream of air, and sterile medium was added to the dried acyl-HSL. All liquid media containing acyl-HSL were prepared immediately prior to use.

\section{Plasmid construction}

We constructed the LuxR expression vector, pLuxR, and the signal response screening plasmid, pluxGFPuv, using the two-plasmid pPROLar.A122 and pPROTet.E133 system from BD Biosciences Clontech (Palo Alto, CA) as plasmid backbones. Plasmid pLuxR encodes LuxR under the control of the hybrid $\mathrm{P}_{\text {lac/ara- } 1}$ promoter and was constructed by cloning PCR-amplified luxR from pKE705 (Egland and Greenberg, 2001), using primers 5'-LuxR (5'-CGAACGGGGTACCCAT GAAAAACATAAATGCCGACGACAC-3') and 3'-LuxR (5'CGTTCGCGGATCCCGTACTTAATTTTTAAAGTATGGGCAA TC-3'), into Kpnl and BamHI digested pPROLar.A122. The sequence of pLuxR, including the promoter and the luxR gene, was verified by sequencing using the upstream primer 5'-LarSeq2 (5'-CCTGAGCAATCACCTATGAACTGTC-3') and internal luxR primer LuxRSeq(int) (5'-CGAAAACATCAG GTCTTATCACTGGG-3'). The pLuxR plasmid contains a kanamycin resistance gene and the $\mathrm{p} 15 \mathrm{~A}$ replication origin. Plasmid pluxGFPuv encodes a variant of green fluorescent protein (GFPuv from pGFPuv, BD Biosciences Clontech, Palo Alto, CA) under the control of the $\mathrm{P}_{l u x \mid}$ promoter. $\mathrm{P}_{l u x l}$ was PCR-amplified from pKE555 (Egland and Greenberg, 1999) using the primers $5^{\prime}$-pluxl (5'-CGAACGCGACGTCAGTC CTTTGATTCTAATAAATTGGATTTTTGTCAC-3') and 3'-pluxl (5'-CTTCTCCTTTACTCATACCAACCTCCCTTGCGTTTATTC $\left.-3^{\prime}\right)$ and gfpuv was PCR-amplified from pGFPuv using $5^{\prime}$ GFPuv (5'-GGGAGGTTGGTATGAGTAAAGGAGAAGAACT TTTCACT-3') and 3'-GFPuv (5'-GTACCCAAGCTTTTATTTG TAGAGCTCATCCATGCCATG-3'). These $\mathrm{P}_{l u x l}$ and gfpuv PCR products were assembled and amplified by PCR with $5^{\prime}$-pluxl and $3^{\prime}$-GFPuv and cloned into pPROTet.E133 between its Aatll and Hindlll sites. The sequence of the entire promoter region and gfpuv gene on pluxGFPuv was verified by DNA sequencing using the primers $5^{\prime}$-pPROTetSeq $\left(5^{\prime}\right.$-CCTCT TACGTGCCGATCAACGTC-3') and GFPuvSeq(int) (5'CGAAGGTTATGTACAGGAACGCAC-3'). This plasmid contains a chloramphenicol resistance gene and the ColE1 replication origin. See Supplementary material, Fig. S3, for plasmid maps of pLuxR and pluxGFPuv. The plasmid encoding the C-terminal fusions of $c-m y c$ with LuxR was cloned using the primers $5^{\prime}$-LuxR and $3^{\prime}$-LuxR-myc (5'-CGTTCGC GGATCCTTACAGATCCTCTTCGCTGATCAGTTTCTGTTCA GCTCCACCATTTTTAAAGTATGGGCAATCAATTG-3') with pLuxR as template. The fusion was cloned into Kpnl and BamHI digested pPROLar.A122 to make pLuxR-myc. Versions of pLuxR-myc encoding the second-generation LuxR variants fused to $c$-myc were cloned similarly to pLuxR-myc using the appropriate pLuxR plasmid encoding the mutant IuxR as template. BamHI, HindlII and Kpnl were purchased from Roche Applied Science (Indianapolis, IN) and Aatll was purchased from New England Biolabs (Beverly, MA).

\section{Library construction and screening}

Error-prone PCR reactions were performed using AmpliTaq DNA polymerase (Applied Biosystems, Foster City, CA) and $50 \mu \mathrm{M} \mathrm{MnCl}{ }_{2}$ to increase the mutation rate as described (Cirino et al., 2003). The primers $5^{\prime}$-luxR and $3^{\prime}$-luxR were used to amplify the luxR gene using pLuxR as the template. The library was constructed by ligating Kpnl and BamHI digested pPROLar.A122 with the products of error-prone PCR using T4 DNA ligase (Invitrogen, Carlsbad, CA). Vent DNA polymerase (New England Biolabs, Beverly, MA) was used to amplify wild-type luxR, which was digested and ligated into pPROLar.A122 for use as a control. The ligation mixtures were transformed into competent $\mathrm{DH} 5 \alpha$ cells harbouring pluxGFPuv and plated onto LB-agar containing the appropriate antibiotics and 50 or $200 \mathrm{nM} \mathrm{C8HSL}$. The plates were incubated at $37^{\circ} \mathrm{C}$ for $18 \mathrm{~h}$ prior to screening. To identify clones of interest, the agar plates were placed over a UVtransilluminator (VWR Scientific, West Chester, PA) at $365 \mathrm{~nm}$ and visually inspected for GFPuv fluorescence. The plates were stored at $4^{\circ} \mathrm{C}$ and re-examined every $2-3 \mathrm{~h}$ until colonies on a control plate expressing wild-type LuxR became visibly fluorescent.

Second-generation libraries were constructed by DNA shuffling as described (Joern, 2003). The primers $5^{\prime}$-luxR and $3^{\prime}$-luxR were used to amplify the mutant luxR genes from six first-generation mutants using $P f u$ Turbo DNA polymerase (Stratagene, La Jolla, CA). After purification and quantification, equal amounts of parent amplification products were mixed and subjected to a DNase I digestion. The $100 \mu \mathrm{l}$ digest contained c. $4 \mu \mathrm{g}$ of the parental mix, $10 \mu \mathrm{l} 0.5 \mathrm{M}$ Tris$\mathrm{HCl}(\mathrm{pH} 7.4), 5 \mu \mathrm{l} 0.2 \mathrm{M}$ manganese chloride and 0.14 units of DNase I. After $1 \mathrm{~min}$ of digestion at $15^{\circ} \mathrm{C}$, the reaction was stopped by adding $5 \mu \mathrm{l} 1 \mathrm{M}$ EDTA and by placing the mixture immediately on ice. The QIAEXII gel extraction kit (Qiagen, Valencia, CA) was used to purify fragments ranging from 50 to $150 \mathrm{bp}$ in length. Fragments were randomly reassembled in a $50 \mu \mathrm{l}$ reaction. Full-length luxR genes were synthesized by diluting the reassembly reaction 50 - to 500 -fold and amplified using Pfu Turbo DNA polymerase and the primers $5^{\prime}$-luxR and $3^{\prime}$-luxR. Ligations and transformations were performed similarly to the first generation and plated onto LB-agar containing $50 \mathrm{nM}$ C8HSL. All 102 colonies that fluoresced after $18 \mathrm{~h}$ were picked, purified and inoculated into $1 \mathrm{ml}$ LB containing the appropriate antibiotics. After $24 \mathrm{~h}$ at $37^{\circ} \mathrm{C}$, the cultures were diluted 10 -fold into fresh LB, and replicastamped using a 96-well pin replicator onto solid media containing 10, 20, 50, 100 and $200 \mathrm{nM} \mathrm{C8HSL}$. Ten mutants that fluoresced in response to 10 or $20 \mathrm{nM} \mathrm{C8HSL}$ were identified for further characterization.

\section{Re-cloning and DNA sequencing}

All mutants identified during the first- and second-generation screens were re-cloned into fresh background plasmids and 
strains to eliminate secondary-site effects. For each mutant, the luxR allele was amplified using Pfu Turbo polymerase and treated with Dpnl. The PCR products were digested and ligated into pPROLar.A122 (as above) and transformed into competent $\mathrm{DH} 5 \alpha$ cells containing pluxGFPuv. The promoter and luxR gene from all mutants of interest were sequenced using the upstream primer $5^{\prime}$-LarSeq2 (5'CCTGAGCAAT CACCTATGAACTGTC-3') and internal lux $R$ primer LuxRSeq(int) (5'-CGAAAACATCAGGTCTTATCACT GGG-3').

\section{Site-directed mutagenesis}

DNA fragments containing each single point mutation of interest were generated using standard methods (Horton et al., 1993). In the first step, the $5^{\prime}$-LuxR primer and a reverse primer containing the desired mutation were used to amplify luxR upstream of the mutation; the $3^{\prime}$-LuxR primer and a forward primer also containing the mutation were used to amplify luxR downstream of the mutation. In the second step, the two overlapping fragments were assembled to yield a fulllength luxR containing a single nucleotide alteration by PCR using the products of step one as template and the $5^{\prime}$-LuxR and $3^{\prime}$-LuxR primer set. These PCR products were digested and ligated into $\mathrm{Kpnl}$ and $\mathrm{BamHI}$ digested pLuxR to replace the wild-type luxR. Sequences of each site-directed mutant were verified by DNA sequencing.

\section{Quantitative characterization of LuxR-mediated gene expression of GFPuv}

The assay used to measure LuxR-mediated gene activation was adapted from a light-based bioassay protocol (Schaefer et al., 2000). Cells were first grown from single colonies or glycerol stocks in LB overnight, then diluted 200-fold into $100 \mathrm{ml}$ of fresh LB medium containing $5 \mathrm{mM}$ potassium phosphate buffer, $\mathrm{pH} 6.5$, and the appropriate antibiotics. Such cultures were incubated with shaking at $37^{\circ} \mathrm{C}$ until they reached an $\mathrm{OD}_{600}$ of 0.5 , and then harvested by centrifugation. Cell pellets were washed and resuspended to an $\mathrm{OD}_{600}$ of 0.6 in bioassay medium $(0.05 \% \mathrm{w} / \mathrm{v}$ tryptone, $0.03 \% \mathrm{v} / \mathrm{v}$ glycerol, $100 \mathrm{mM}$ sodium chloride, $50 \mathrm{mM}$ magnesium sulphate and $5 \mathrm{mM}$ potassium phosphate buffer, $\mathrm{pH} 6.5$, containing antibiotics. The suspension was subsequently transferred into 48-well plates (VWR International, cat. no. 82004-674) containing $0.5 \mathrm{ml}$ of bioassay medium with acyl-HSL, to a total volume of $2.5 \mathrm{ml}$ per well. Thereafter, the 48 -well plates were shaken at $37^{\circ} \mathrm{C}$ for $4 \mathrm{~h}$. From each well, $200 \mu \mathrm{l}$ was transferred to wells of a white 96-well microplate with a clear bottom. GFPuv fluorescence (395 nm excitation, $509 \mathrm{~nm}$ emission, $495 \mathrm{~nm}$ cutoff) was measured using a fluorescence microtiterplate reader (Molecular Devices, SpectraMAX Gemini XS); cell densities were measured using a microtiterplate reader at $600 \mathrm{~nm}$. Fluorescence by cell suspensions was normalized to optical density. The fluorescence output of pPROLar.A122 with pluxGFPuv was used to determine the background fluorescence without LuxR, and this background fluorescence value was subtracted from all fluorescence measurements obtained with wild-type pLuxR and all mutants to determine fluorescence only due to LuxR-dependent gene activation. For a given variant, the same batch of cells was used for comparing responses to (i) different concentrations of a given acyl-HSL, or (ii) different acyl-HSLs.

\section{Assays of LuxR abundance in vivo by Western immunodetection}

To estimate the relative concentrations of LuxR in uninduced and fully induced second-generation variants, we constructed C-terminal LuxR fusions to the amino acid sequence GGAEQKLISEEDL, i.e. the $c-m y c$ epitope tag with an Nterminal GGA linker. pLuxR-myc was transformed into $\mathrm{DH} 5 \alpha$ (pluxGFPuv); the protein it encodes was determined to function similarly to its parent LuxR without the tag. Strains expressing the C-terminal c-myc fusions of wild-type LuxR and the second-generation LuxR variants were grown, harvested, resuspended and incubated in the absence of acyl$\mathrm{HSL}$ or with $1 \mu \mathrm{M} 3 \mathrm{OC} 6 \mathrm{HSL}$ in a similar manner as during the quantitative characterization of LuxR-mediated gene expression of GFPuv (see above). After incubation in the presence or absence of acyl-HSL and after monitoring $\mathrm{OD}_{600}$ and fluorescence: $1 \mathrm{ml}$ of each cell suspension of interest was resuspended in $50 \mu \mathrm{l}$ of SDS loading buffer. Samples were boiled for $5 \mathrm{~min}$ and centrifuged for $10 \mathrm{~min}$ before the proteins in the clarified fluid were size fractionated via standard SDS-PAGE. Proteins were electrotransferred to nitrocellulose membranes (Invitrogen). After an $8 \mathrm{~h}$ blocking step using tris-buffered saline (TBS; $50 \mathrm{mM}$ Tris- $\mathrm{HCl}, 150 \mathrm{mM}$ $\mathrm{NaCl}, \mathrm{pH} 7.5)$ containing $5 \%$ non-fat dried milk, membranes were incubated for $8 \mathrm{~h}$ in TBS buffer containing $1 \%$ non-fat dried milk and $4 \%$ bovine serum albumin (BSA, Sigma) with anti-myc mouse antisera (Invitrogen). Antibody-protein complexes were visualized using horseradish peroxidase-coupled anti-murine second antibody (Invitrogen) and SuperSignal West Pico Substrate (Pierce, Rockford, IL). Chemiluminescence was detected using Kodak BioMax Light Film (Rochester, NY). The developed film was subsequently scanned using a Hewlett Packard Scanjet 4400c and HP Precisionscan Pro software (version 3.1). Protein concentrations, relative to wild-type LuxR, were determined from the scanned image via densitometry analysis using Quantity One software (Bio-rad, version 4.1.1).

\section{Acknowledgements}

We are grateful to Bernard Hauer for providing synthetic C5HSL. We thank M. Barnet, T. Ciche, J. Gralnick, J. Silberg, M. Urbanowski and L. You for critical reading of the manuscript. This research was supported by the Defense Advanced Research Projects Agency (DARPA) grant N66001-02-8929 and by National Science Foundation, Biological Information Technology and Storage (BITS) grant EIA-0130613. Disclaimer: Any opinions, findings and conclusions or recommendations expressed in this publication are those of the author(s) and do not necessarily reflect the views of the DARPA.

\section{Supplementary material}

The following material is available from http://www.blackwellpublishing.com/products/journals/ suppmat $/ \mathrm{mmi} / \mathrm{mmi} 4437 / \mathrm{mmi} 4437 \mathrm{sm} . \mathrm{htm}$ 
Fig. S1. Mutations in LuxR responsible for altering acylHSL specificity differ from those which result in a loss of function.

Fig. S2. A. Multiple sequence alignments of 19 members of the LuxR family of transcriptional regulators.

B. List of LuxR homologues used in the multiple sequence alignment, including the source species and the acyl-HSLs to which they are reported to respond preferentially and strongly.

Fig. S3. Plasmids employed for screening libraries of luxR mutants.

Table S1. Nucleotide and amino acid changes in the recovered IuxR/LuxR mutants.

\section{References}

Bainton, N.J., Stead, P., Chhabra, S.R., Bycroft, B.W., Salmond, G.P.C., Stewart, G., and Williams, P. (1992) N(3-Oxohexanoyl)-L-homoserine lactone regulates carbapenem antibiotic production in Erwinia carotovora. Biochem J 288: 997-1004.

von Bodman, S.B., and Farrand, S.K. (1995) Capsular polysaccharide biosynthesis and pathogenicity in Erwinia stewartii require induction by an $\mathrm{N}$-acyl-homoserine lactone autoinducer. J Bacteriol 177: 5000-5008.

Chai, Y.R., and Winans, S.C. (2004) Site-directed mutagenesis of a LuxR-type quorum-sensing transcription factor: alteration of autoinducer specificity. Mol Microbiol 51: 765776.

Chugani, S.A., Whiteley, M., Lee, K.M., D’Argenio, D., Manoil, C., and Greenberg, E.P. (2001) QscR, a modulator of quorum-sensing signal synthesis and virulence in Pseudomonas aeruginosa. Proc Natl Acad Sci USA 98: 2752-2757.

Cirino, P.C., Mayer, K.M., and Umeno, D. (2003) Generating libraries using error-prone PCR. In Directed Evolution Library Creation. Arnold, F.H., and Georgiou, G. (eds). Totawa, NJ: Humana Press, pp. 3-9.

Crameri, A., Whitehorn, E.A., Tate, E., and Stemmer, W.P.C. (1996) Improved green fluorescent protein by molecular evolution using DNA shuffling. Nat Biotechnol 14: 315319.

Davies, D.G., Parsek, M.R., Pearson, J.P., Iglewski, B.H., Costerton, J.W., and Greenberg, E.P. (1998) The involvement of cell-to-cell signals in the development of a bacterial biofilm. Science 280: 295-298.

Eberhard, A., Widrig, C.A., McBath, P., and Schineller, J.B. (1986) Analogs of the autoinducer of bioluminescence in Vibrio fischeri. Arch Microbiol 146: 35-40.

Egland, K.A., and Greenberg, E.P. (1999) Quorum sensing in Vibrio fischeri: elements of the Iux/ promoter. Mol Microbiol 31: 1197-1204.

Egland, K.A., and Greenberg, E.P. (2001) Quorum sensing in Vibrio fischeri: analysis of the LuxR DNA binding region by alanine-scanning mutagenesis. J Bacteriol 183: 382386.

Engebrecht, J., and Silverman, M. (1984) Identification of genes and gene products necessary for bacterial bioluminescence. Proc Natl Acad Sci USA 81: 4154-4158.

Fuqua, W.C., and Winans, S.C. (1994) A LuxR-Luxl type regulatory system activates agrobacterium Ti plasmid con- jugal transfer in the presence of a plant tumor metabolite. $J$ Bacteriol 176: 2796-2806.

Fuqua, W.C., Winans, S.C., and Greenberg, E.P. (1994) Quorum sensing in bacteria - the LuxR-Luxl family of cell density-responsive transcriptional regulators. J Bacteriol 176: 269-275.

Fuqua, C., Parsek, M.R., and Greenberg, E.P. (2001) Regulation of gene expression by cell-to-cell communication: Acyl-homoserine lactone quorum sensing. Annu Rev Genet 35: 439-468.

Gambello, M.J., and Iglewski, B.H. (1991) Cloning and characterization of the Pseudomonas aeruginosa LasR gene, a transcriptional activator of elastase expression. $J$ Bacteriol 173: 3000-3009.

Horton, R.M., Ho, S.N., Pullen, J.K., Hunt, H.D., Cai, Z.L. and Pease, L.R. (1993) Gene-splicing by overlap extension. Methods Enzymol 217: 270-279.

Jenson, R.A. (1976) Enzyme recruitment in evolution of new function. Annu Rev Microbiol 30: 409-425.

Joern, J.M. (2003) DNA shuffling. In Directed Evolution Library Creation. Arnold, F.H., and Georgiou, G. (eds). Totawa, NJ: Humana Press, pp. 85-89.

Kacser, H., and Beeby, R. (1984) Evolution of catalytic proteins or on the origin of enzyme species by means of natural selection. $J$ Mol Evol 20: 38-51.

Kaplan, H.B., and Greenberg, E.P. (1985) Diffusion of autoinducer is involved in regulation of the Vibrio fischeri luminescence system. J Bacteriol 163: 1210-1214.

Lazcano, A., Diazvillagomez, E., Mills, T., and Oro, J. (1994) On the levels of enzymatic substrate-specificity - implications for the early evolution of metabolic pathways. Adv Space Res 15: 345-356.

Lerat, E., and Moran, N.A. (2004) The evolutionary history of quorum-sensing systems in bacteria. Mol Biol Evol 21: 903-913.

Matsumura, I., and Ellington, A.D. (2001) In vitro evolution of $\beta$-glucuronidase into a $\beta$-galactosidase proceeds through non-specific intermediates. J Mol Biol 305: 331339.

Miller, M.B., and Bassler, B.L. (2001) Quorum sensing in bacteria. Annu Rev Microbiol 55: 165-199.

Parsek, M.R., and Greenberg, E.P. (1999) Quorum sensing signals in development of Pseudomonas aeruginosa biofilms. Methods Enzymol 310: 43-55.

Passador, L., Cook, J.M., Gambello, M.J., Rust, L., and Iglewski, B.H. (1993) Expression of Pseudomonas aeruginosa virulence genes requires cell-to-cell communication. Science 260: 1127-1130.

Pearson, J.P., Van Delden, C., and Iglewski, B.H. (1999) Active efflux and diffusion are involved in transport of Pseudomonas aeruginosa cell-to-cell signals. J Bacteriol 181: 1203-1210.

Pierson, L.S., Keppenne, V.D., and Wood, D.W. (1994) Phenazine antibiotic biosynthesis in Pseudomonas aureofaciens $30-84$ is regulated by $\mathrm{PhzR}$ in response to cell density. J Bacteriol 176: 3966-3974.

Redfield, R.J. (2002) Is quorum sensing a side effect of diffusion sensing? Trends Microbiol 10: 365-370.

Santoro, S.W., and Schultz, P.G. (2002) Directed evolution of the site specificity of Cre recombinase. Proc Natl Acad Sci USA 99: 4185-4190. 
Schaefer, A.L., Hanzelka, B.L., Eberhard, A., and Greenberg, E.P. (1996) Quorum sensing in Vibrio fischeri: Probing autoinducer-LuxR interactions with autoinducer analogs. $J$ Bacteriol 178: 2897-2901.

Schaefer, A.L., Hanzelka, B.L., Parsek, M.R., and Greenberg, E.P. (2000) Detection, purification, and structural elucidation of the acyl-homoserine lactone inducer of Vibrio fischeri luminescence and other related molecules. Methods Enzymol 305: 288-301.

Schaefer, A.L., Taylor, T.A., Beatty, J.T., and Greenberg, E.P. (2002) Long-chain acyl-homoserine lactone quorumsensing regulation of Rhodobacter capsulatus gene transfer agent production. J Bacteriol 184: 6515-6521.

Shadel, G.S., Young, R., and Baldwin, T.O. (1990) Use of regulated cell-lysis in a lethal genetic selection in Escherichia coli - identification of the autoinducer-binding region of the LuxR protein from Vibrio fischeri ATCC-7744. J Bacteriol 172: 3980-3987.

Slock, J., Vanriet, D., Kolibachuk, D., and Greenberg, E.P. (1990) Critical regions of the Vibrio fischeri LuxR protein defined by mutational analysis. J Bacteriol 172: 39743979.

Stevens, A.M., and Greenberg, E.P. (1997) Quorum sensing in Vibrio fischeri: essential elements for activation of the luminescence genes. J Bacteriol 179: 557-562.

Stevens, A.M., and Greenberg, E.P. (1999) Transcriptional activation by LuxR. In Cell-Cell Signaling in Bacteria. Dunny, G.M., and Winans, S.C. (eds). Washington, DC: American Society for Microbiology Press, pp. 231242.

Stevens, A.M., Fujita, N., Ishihama, A., and Greenberg, E.P.
(1999) Involvement of the RNA polymerase alpha-subunit C-terminal domain in LuxR-dependent activation of the Vibrio fischeri luminescence genes. J Bacteriol 181: 47044707.

Urbanowski, A.L., Lostroh, C.P., and Greenberg, E.P. (2004) Reversible acyl-homoserine lactone binding to purified Vibrio fischeri LuxR protein. J Bacteriol 186: 631-637.

Voigt, C.A., Mayo, S.L., Arnold, F.H., and Wang, Z.G. (2001) Computational method to reduce the search space for directed protein evolution. Proc Natl Acad Sci USA 98: 3778-3783.

Voziyanov, Y., Stewart, A.F., and Jayaram, M. (2002) A dual reporter screening system identifies the amino acid at position 82 in Flp site-specific recombinase as a determinant for target specificity. Nucleic Acids Res 30: 1656-1663.

Waley, S.G. (1969) Some aspects of evolution of metabolic pathways. Comp Biochem Physiol 30: 1-11.

Whiteley, M., Lee, K.M., and Greenberg, E.P. (1999) Identification of genes controlled by quorum sensing in Pseudomonas aeruginosa. Proc Natl Acad Sci USA 96: 13904-13909.

Ycas, M. (1974) On earlier states of the biochemical system. $J$ Theor Biol 44: 145-160.

Zhang, R.G., Pappas, T., Brace, J.L., Miller, P.C., Oulmassov, T., Molyneauz, J.M., et al. (2002) Structure of a bacterial quorum-sensing transcription factor complexed with pheromone and DNA. Nature 417: 971-974.

Zhu, J., and Winans, S.C. (2001) The quorum-sensing transcriptional regulator TraR requires its cognate signaling ligand for protein folding, protease resistance, and dimerization. Proc Natl Acad Sci USA 98: 1507-1512. 